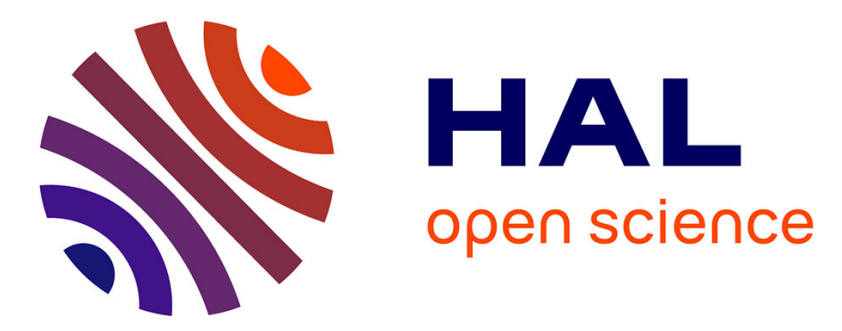

\title{
Indium segregation and misorientation effects on the optical properties of MBE grown In0.35Ga0.65As/GaAs quantum wells
}

C. Monier, Joël Leymarie, A. Marti Ceshin, N. Grandjean, Aime Vasson, M. Leroux, J. Massies

\section{To cite this version:}

C. Monier, Joël Leymarie, A. Marti Ceshin, N. Grandjean, Aime Vasson, et al.. Indium segregation and misorientation effects on the optical properties of MBE grown In0.35Ga0.65As/GaAs quantum wells. Journal de Physique IV Proceedings, 1993, 03 (C5), pp.C5-295-C5-298. 10.1051/jp4:1993559 . jpa-00251645

\section{HAL Id: jpa-00251645 https://hal.science/jpa-00251645}

Submitted on 1 Jan 1993

HAL is a multi-disciplinary open access archive for the deposit and dissemination of scientific research documents, whether they are published or not. The documents may come from teaching and research institutions in France or abroad, or from public or private research centers.
L'archive ouverte pluridisciplinaire HAL, est destinée au dépôt et à la diffusion de documents scientifiques de niveau recherche, publiés ou non, émanant des établissements d'enseignement et de recherche français ou étrangers, des laboratoires publics ou privés. 


\title{
Indium segregation and misorientation effects on the optical properties of $\mathrm{MBE}$ grown $\mathrm{In}_{0.35} \mathrm{Ga}_{0.65} \mathrm{As} / \mathrm{GaAs}$ quantum wells
}

\author{
C. MONIER, J. LEYMARIE, A. MARTI CESHIN*, N. GRANDJEAN*, A. VASSON, \\ A.-M. VASSON, M. LEROUX* ${ }^{*}$ and J. MASSIES*
}

Laboratoire de Physique des Milieux Condensés, Unité de Recherche Associée au Centre National de la Recherche Scientifique 796, Université Blaise Pascal Clermont II, 24 avenue des Landais, 63177 Aubière cedex, France

* Laboratoire de Physique du Solide et Energie Solaire, Centre National de la Recherche Scientifique, rue Bernard Grégory, 06560 Valbonne, France

\begin{abstract}
Photoluminescence (PL), reflectivity and thermally-detected optical absorption (TDOA) experiments have been carried out, at liquid helium temperatures, on $\mathrm{In}_{0.35} \mathrm{Ga}_{0.65} \mathrm{As} / \mathrm{GaAs}$ quantum wells (QWs) with different thicknesses of 4, 6, 8 and 10 monolayers (MLs), grown by molecular beam epitaxy (MBE) on substrates oriented exactly on (100) (nominal growth) or misoriented by $4^{\circ}$ or $6^{\circ}$ towards $(111)_{\mathrm{Ga}}$ (vicinal growth). Indium segregation effects are considered in the comparison of the calculated fundamental $\left(e_{1} h_{1}\right)$ transition energies of the QWs with the experimental results. Within the framework of a simple model, a segregation energy of $0.35 \mathrm{eV}$ is used to fit the experimental data. It is shown that the misorientation improves the optical properties of QWs: diminution of the spectral broadenings and reduction of the blue shift between reflectivity structures or TDOA peaks and luminescence lines. This suggests a better quality of the interfaces and an improvement of the strain homogeneity. A step edge effect on the fundamental transition energy of the 6 and $8 \mathrm{ML}$ quantum wells is shown but the blue shift observed for a growth on a $4^{\circ}$ off misoriented surface can also be due to a modification of the growth mode.
\end{abstract}

\section{Introduction}

It is known that the growth of strained (In,Ga)As layers on a misoriented GaAs substrate improves their structural or optical properties such as the reduction of the strain inhomogeneity and composition fluctuations or the diminution of the emission linewidths [1]. The surface segregation of In, which occurs during the growth of (In,Ga)As quantum wells (QWs) by molecular beam epitaxy (MBE), modifies the In concentration profile of the well and has to be taken into account to compare the calculated transition energies with those deduced from spectroscopy experiments [2,3]. Moreover, it has been shown that the degradation of the optical properties of MBE grown (In,Ga)As/GaAs QW's is correlated with the strain-induced 2D-3D growth mode transition [4]. In this work, the effects of In segregation and substrate misorientation on the optical properties of $\mathrm{MBE}$ grown $\operatorname{In}_{0.35} \mathrm{Ga}_{0.65} \mathrm{As} / \mathrm{GaAs}$ $\mathrm{QWs}$ are investigated. The influence of the misorientated surface on the growth process and on the energy levels of the wells is discussed through the experimental results.

\section{Growth and experimental details}

The In $0.35 \mathrm{Ga}_{0.65} \mathrm{As} / \mathrm{GaAs}$ quantum wells have been grown by molecular beam epitaxy at a growth temperature $\left(\mathrm{T}_{\text {growth }}\right)$ of $540^{\circ} \mathrm{C}$ on $n+$ doped GaAs substrates oriented in the exact (001) plane (nominal growth) or misoriented by $4^{\circ}$ or $6^{\circ}$ towards $(111)_{\mathrm{Ga}}$ (vicinal growth). Two series of QWs with thickness between 2 and 10 monolayers (MLs) were achieved by introducing simultaneously exactly oriented and misoriented substrates in the MBE system.

Reflectivity and photoluminescence ( $\mathrm{PL}$ ) experiments have been performed at $2 \mathrm{~K}$. Thermallydetected optical absorption (TDOA) has been carried out at $0.35 \mathrm{~K}$ [5]. It must be recalled that, in this non conventional technique, the non radiative de-excitation is observed via the heating of the sample [6].

\section{Low temperature photoluminescence spectra}

Photoluminescence spectra of 4,6,8 and $10 \mathrm{ML}$ quantum wells grown in a first run on nominal (sample 978 ) and $4^{\circ}$ and $6^{\circ}$ misoriented substrates (samples 981 and 983) are displayed in figure 1 . The luminescence of the $4 \mathrm{ML} \mathrm{QW}$ is absent in the spectra. In order to avoid any confusion 
about the correspondence of particular PL lines with the QW thicknesses, it is worth to precise that the growth of a nominal $6 \mathrm{ML}$ single $\mathrm{QW}$ gives the PL energy corresponding to that of the $6 \mathrm{ML} \mathrm{QWs}$ of the samples elaborated on nominal surface and presented here. Concerning the 6 and $8 \mathrm{ML}$ QWs, an increase of the luminescence energy (20-30 meV) is observed for the samples grown on vicinal substrates. Together with the blue shift, a reduction by a factor 2-3 of the full width at half maximum (FWHM) of the PL lines is clearly in evidence. If the linewidth can be analysed in terms of homogeneity of interfaces, it can be concluded that these narrow emissions reveal smooth interfaces. However, it would be premature to deduce that vicinal growth induces such a shift of the mean energy for each well. Indeed, the luminescence does not reflect the density of excitonic states because of the localisation of excitons on low energy states. As an example, it is shown for sample 981 ( $4^{\circ}$ off) that the luminescence maximum of the $6 \mathrm{ML}$ and $8 \mathrm{ML}$ QWs is shift by about $2 \mathrm{meV}$ towards the high energy side when the excitation intensity

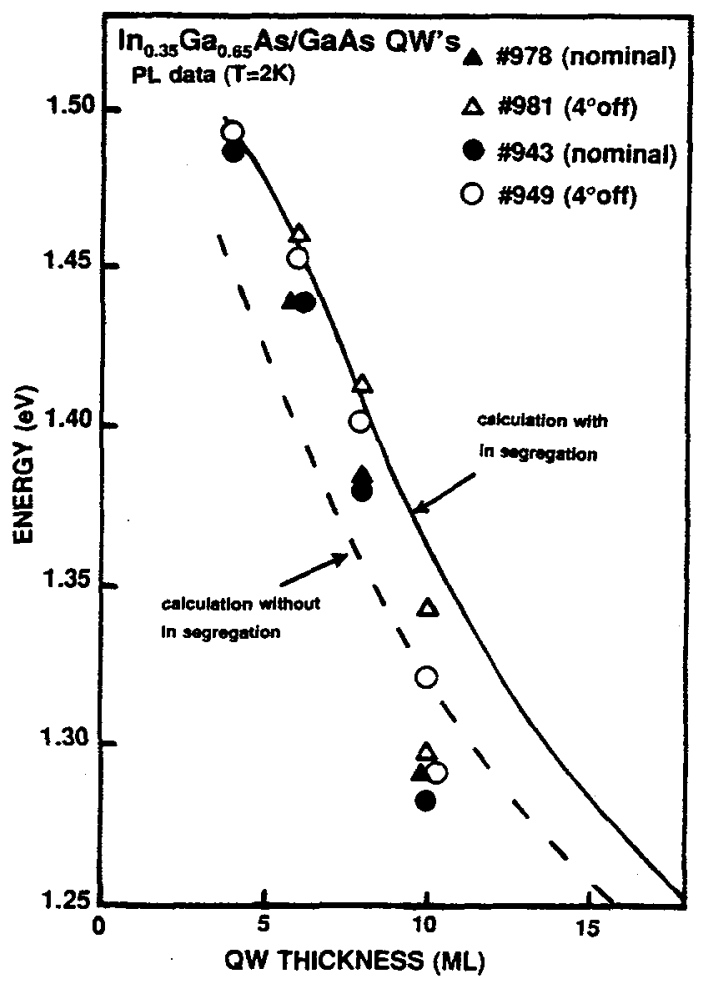

Fig. 2: $P L$ energy versus $Q W$ thickness. The solid line (dashed line) represents the calculated $\mathrm{e}_{1} \mathrm{hh}_{1}$ energy by taking into account (neglecting) the In surface segregation.

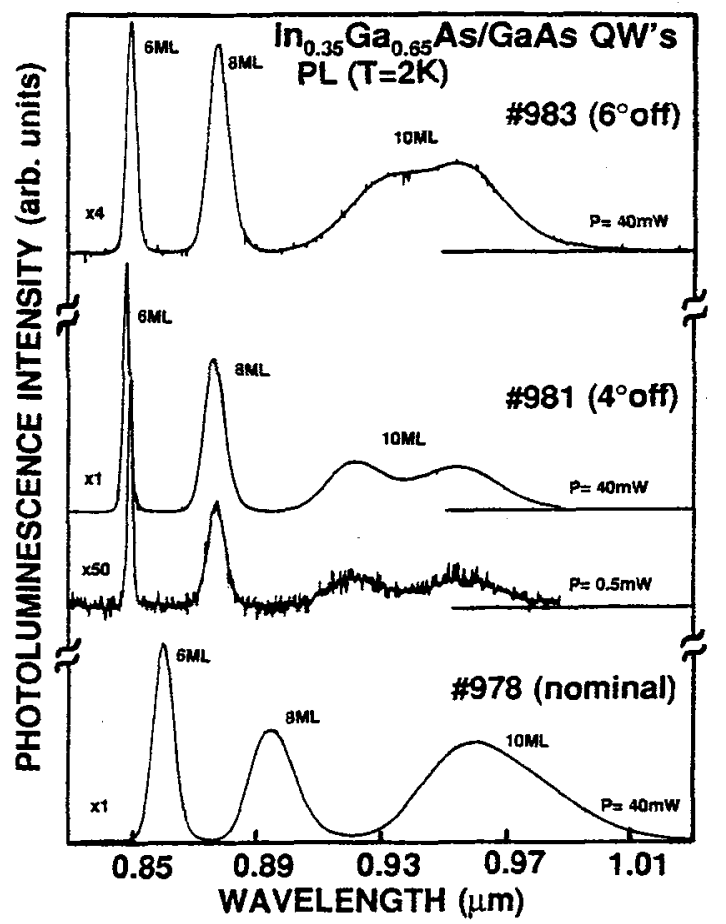

Fig. 1: PL spectra of samples grown on nominal and misoriented substrates (first run).

increases by two orders of magnitude. The emission of the $10 \mathrm{ML}$ QW grown on vicinal surfaces is split into two components as discusssed below.

As reflectivity features are not detected in any of the samples investigated, the PL energies of the QWs are reported in figure 2 and compared with calculations including In segregation effects. A simple mass-action law involving a phenomenological segregation energy $E_{s}$ is used to take into account the fact that In atoms segregate to the surface during the growth so that the indium concentration profile of the quantum well can be evaluated [3]. The energy of the electron-heavy hole fundamental transition $\left(e_{1} h h_{1}\right)$ is then calculated within the transfer matrix formalism including the strain effects on the $\mathrm{In}_{0.35} \mathrm{Ga}_{0.65}$ As band structure. An overall agreement is obtained with an $E_{s}$ value of 0.35 $\mathrm{eV}$ and a strained valence band ratio $\left(Q_{v}=\Delta E_{v} / \Delta E_{g}\right)$ of 0.3 . An exciton binding energy of $7 \mathrm{meV}$ is included in the present calculation. The segregation energy determined for thenominal indium concentration $(x)$ of 0.35 has to be compared with the value of $0.22 \mathrm{eV}$ found by Nagle et al for $x=0.2\left(\mathrm{~T}_{\text {growth }}=560^{\circ} \mathrm{C}\right)$ [3]. 


\section{Reflectivity and TDOA spectra}

Reflectivity and TDOA spectra with the corresponding PL spectrum of two samples grown in a second run are shown in figure 3. Five quantum wells of $2,4,6,8$ and $10 \mathrm{ML}$ thickness were grown on nominal and $4^{\circ}$ off misoriented substrates (samples 943 and 949). Since the luminescence of the thinnest $\mathrm{QW}$ is not detected, as in the first set of samples, it will not be the subject of the discussion. In agreement with the luminescence, the reflectivity structures are narrower for the QWs grown on vicinal surface than in the nominal case; this indicates a better quality of the interfaces. Moreover, for the two samples, the degradation of the interfaces with the increase of the QW thickness induces a broadening and a weakening of the reflectivity structures so that the reflectivity of the $8 \mathrm{MLs}$ QW (nominal growth) is not detected. It has to be noted that the PL peaks lie at lower energy than the energy positions of the reflectivity and TDOA structures. This is due to the localisation of excitons on low energy states within the well. Table 1 gives the blue shift between TDOA and photoluminescence together with the FWHM of the of PL lines. The linewidth and the energy of the peaks depend slightly upon the excitation intensity and are also given for a laser intensity of approximately $10 \mathrm{~W} \cdot \mathrm{cm}^{-2}(\mathrm{P}=40 \mathrm{~mW})$.

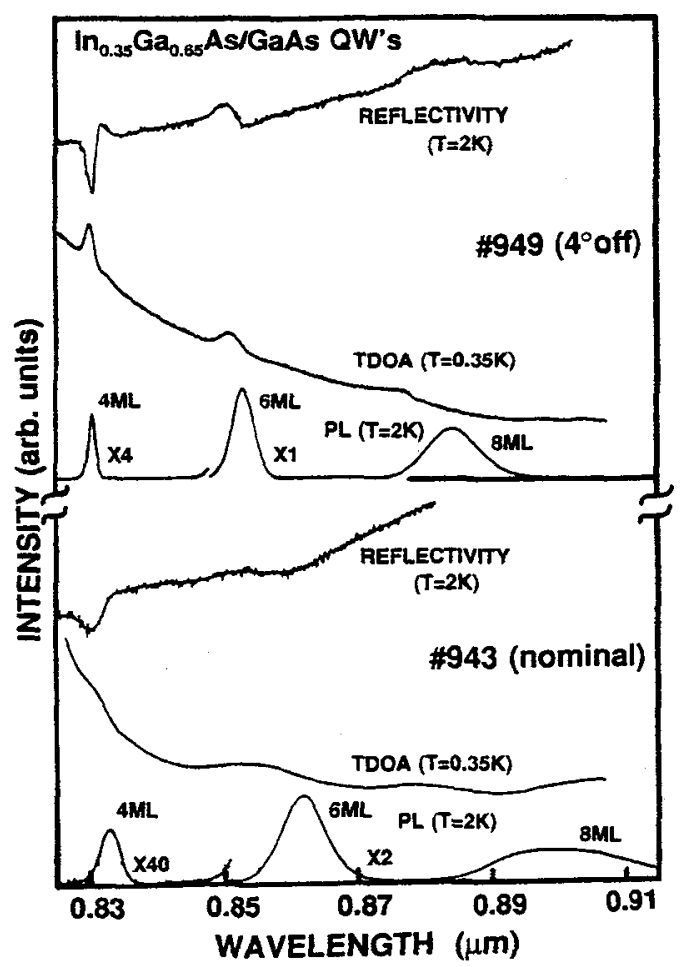

Fig. 3: PL, reflectivity and TDOA spectra of samples from the second run.

\begin{tabular}{|c|c|c|c|c|c|c|}
\hline \multirow{2}{*}{ Layer thickness } & \multicolumn{2}{|c|}{$4 \mathrm{ML}$} & \multicolumn{2}{c|}{$6 \mathrm{ML}$} & \multicolumn{2}{c|}{$8 \mathrm{ML}$} \\
\cline { 2 - 7 } & nominal & $4^{\circ}$ off & nominal & $4^{\circ}$ off & nominal & $4^{\circ}$ off \\
\hline Blue shift (meV) & 4 & $\approx 0$ & 10 & 4 & 28 & 13 \\
\hline FWHM (meV) & 6.2 & 2.1 & 12.6 & 6.6 & 34.2 & 14.4 \\
\hline
\end{tabular}

Table 1: Blue shift between TDOA and photoluminescence (samples 943 and 949) and PL linewidths under an excitation intensity of about $10 \mathrm{~W} . \mathrm{cm}^{-2}$.

\section{Discussion}

When a reasonable agreement is found between PL data and calculations for $Q W$ thicknesses between 4 to $8 \mathrm{MLs}$, the luminescence energy drops for the $10 \mathrm{ML}$ quantum well and a double structure appears in the vicinal growth case (figure 2). A first possible explanation is to consider the strain relaxation process of the wider well which induces a diminution of the transition energy together with a broadenning of the PL line. The two broad peaks related to misoriented samples are then explained by the existence of regions completely relaxed and partially strained. However, the presence of dislocations which release the strain induces a decrease of the emission intensity. It is not the case in all the investigated samples where the integrated intensity of the $10 \mathrm{ML} \mathrm{QW}$ is greater than the intensity of the others. On the other hand, transmission electron microscopy (TEM) analyses have revealed that, on similar samples, dislocations appear only up to about 22 MLs [7]. Consequently, it must be concluded that the high energy peak corresponds roughly to the nominal thickness of the well (10 MLs) and that the low energy band emission comes from islands of 14-16 ML thickness deduced from the calculated curve. As no significant change in the relative PL intensity of the two lines occurs under various excitation intensities, the density of islands appears to be comparable to the density of nominal thickness regions. Considering more generally the whole set of the grown wells, it can be concluded that the use of misoriented substrates limits the degradation of the optical properties which begins to appear for a thickness of $8 \mathrm{MLs}$ in the case of nominal growth. The similar behaviour of the $4^{\circ}$ off and $6^{\circ}$ off samples does not allow to deduce an optimal angle for the misorientation. 
From a growth point of view, the island formation proves that the growth mode is tri-dimensional (3D). The PL data enable to consider that the two-dimensional (2D) growth mode of $\operatorname{In}_{0.35} \mathrm{Ga}_{0.65} \mathrm{As}$ on GaAs persists up to $8 \mathrm{MLs}$. This result is in agreement with the determination of the 2D-3D growth mode transition threshold deduced from reflection high energy electron diffraction (RHEED) experiments carried out during the epitaxy on a nominal surface [4]. However, the misorientation seems to slightly increase the threshold. A qualitative explanation can be found by comparing the diffusion length of the adatoms on the surface with the length of the terraces induced by the misorientation. If the diffusion length is greater than the terrace length, the atoms can diffuse to the step edge and then contribute to a 2D growth even if the conditions of such a growth mode are not favorable. Before the 2D-3D growth mode transition, the better control of the 2D growth mode induced by the terraces leads to a weaker roughness of the interfaces and to a better homogeneity of the strain. The effect on the optical properties is a reduction in the spectral broadening of the PL lines or reflectivity and TDOA structures.

The effect of the step on the energy of the electronic states of the well within the assumption of an ideal well is now considered. At the edge of the step, the strain state of the well cannot be described by a biaxial deformation and the local deformation of the barrier material (GaAs) has to be evaluated in order to quantify accurately the energies of the levels near the step edge of the quantum well via deformation potential theory. Moreover, it has been stressed that in the well-known GaAs/(Ga,Al)As system a thickness discontinuity of one monolayer exists at the step edge if a regular array of steps at the two interfaces of the QW is considered and a simple approach has been proposed to evaluate the blue shift of the fundamental transition [8]. The local perturbation at the step edge, compared with the large lateral exciton extension $(\approx 100 \AA)$, suggests that the energy shift is small. It seems that the absorption line energy of $4 \mathrm{ML}$ QW grown on $4^{\circ}$ off misoriented substrate lies slightly at higher energy than that related to the nominal growth. However, the blue shift, if it exists, does not exceed $1 \mathrm{meV}$. On an other hand, the blue shift between misoriented and nominal samples for the 6 and $8 \mathrm{ML}$ quantum wells is about 8 and $4 \mathrm{meV}$ respectively. Thus, a step effect on the $e_{1} h_{1}$ energy is evidenced.

\section{Conclusion}

Indium segregation effects play a significant role on the energy levels of (In,Ga)As/GaAs QWs and have to be taken into account for the comparison of the experimental data with the calculations. The segregation energy deduced for an In concentration of 0.35 within the framework of a simple model is greater than that determined in the case of $\mathrm{In}_{0.2} \mathrm{Ga}_{0.8} \mathrm{As} / \mathrm{GaAs} \mathrm{QWs}$ by other authors. The increase of the In segregation can be due to the increase of the strain. Concerning the optical properties of QWs grown on nominal and misoriented ( $4^{\circ}$ and $6^{\circ}$ off) substrate, it is shown that the misorientation reduces the spectral broadening of the luminescence lines, the reflectivity structures and the TDOA peaks. This suggests a better quality of interfaces and an improvement of the strain homogeneity. It can be deduced that $2 \mathrm{D}$ growth is kept for a wider QW thickness in the case of a growth on a misoriented surface. A step edge effect on the fundamental transition energy of the 6 and $8 \mathrm{ML}$ quantum wells is in evidence but the blue shift observed for a growth on a $4^{\circ}$ off misoriented surface can be also attributed to a modification of the growth mode.

\section{Acknowledgements} discussions.

The authors are grateful to Dr G. Neu for his interest in this work and to P. Disseix for helpful

\section{References:}

[1] Morris D., Roth A.P., Masut R.A., Lacelle C. and Brebner J.L., J. Appl. Phys. 64 (1988) 4135-4139.

[2] Muraki K., Fukatsu S., Shiraki Y. and Ito R., Appl. Phys. Lett. 6 (1992) 557-559 and J. Crystal Growth 127 (1993) 546-549.

[3] Nagle J., Landesman J.P., Larive M., Mottet C. and Bois P., J. Crystal Growth 127 (1993) $550-554$.

[4] Marti Ceschin A. and Massies J., J. Crystal Growth 114 (1991) 693-699.

[5] Boffety D., Leymarie J., Vasson A., Vasson A.-M., Bates C.A., Chamberlain J.M., Dunn J.L., Heinini M. and Hughes O.H., Semicond. Sci. Technol. 8 (1993) 1408-1411.

[6] Vasson A.-M., Vasson A., Leymarie J., Disseix P., Boring P. and Gil B., Semicond. Sci. Technol. 8 (1993) 303-306.

[7] Delamarre $\mathrm{C}$, private communication.

[8] Chastaing B., Leroux M., Neu G., Grandjean N., Deparis C. and Massies J., Phys. Rev. B, 47 (1993) 1292-1298. 